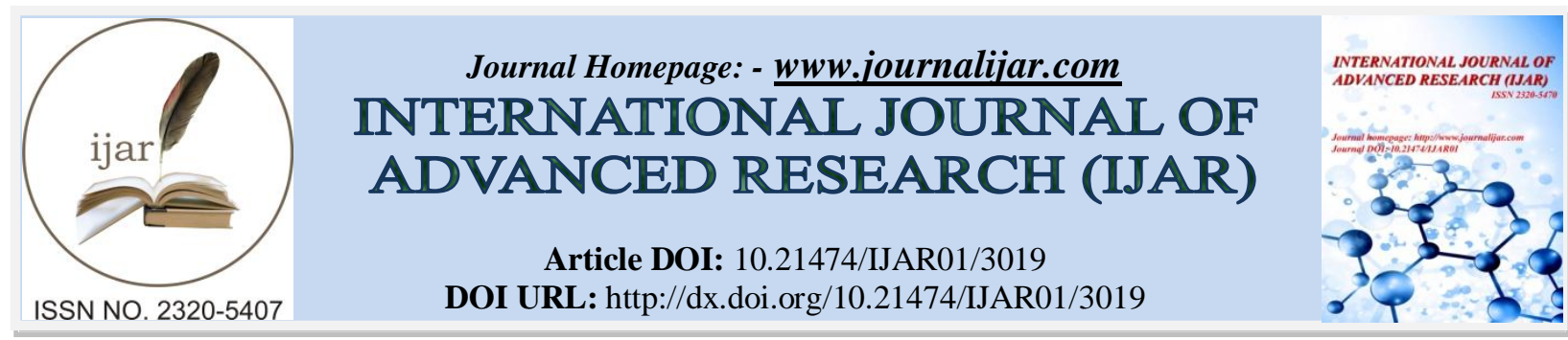

RESEARCH ARTICLE

\title{
THE INCIDENCE OF CHOLELITHIASIS AFTER CORRECTIVE WEIGHT LOSS SURGERY
}

\author{
Ahmed Hamouda Arnaout ${ }^{1}$ and Mahmoud Abdel Latif Bahram ${ }^{2}$. \\ 1. Department of Internal Medicine, Faculty of Medicine, Zagazig University, Egypt. \\ 2. Department of Surgery, Faculty of Medicine, Menoufia University, Egypt.
}

\section{Manuscript Info}

Manuscript History

Received: 29 November 2016

Final Accepted: 27 December 2016

Published: January 2017

Key words:-

Cholelithiasis, sleeve gastrectomy, Roux en $\mathrm{Y}$ gastric bypass.

\begin{abstract}
Introduction: Morbid obesity is a worldwide health problem; cholelithiasis considered the third most common disease between obese patients. Of $30-40 \%$ of patients undergone corrective weight loss surgery developed cholelithiasis shortly after the surgery.

Aim of the study: to detect the relation and incidence of cholelithiasis those necessitate cholecystectomy after corrective weight loss surgery (sleeve gastrectomy and roux en Y gastric bypass) during a one year follow up and the necessary of prior cholecystectomy for those patients. Patients and methods: Morbid obesity patients underwent corrective weight loss surgery (sleeve gastrectomy and roux en Y gastric bypass) in the period from June 2014 till June 2016, patients with unremarkable study at abdominal ultrasound before the surgery and patient with one year follow up after the surgery included in the study. Two comparative groups of patients were done: group A who underwent laparoscopic sleeve gastrectomy and group B who had laparoscopic roux en Y gastric bypass. The primary outcome measure was gallstones or mud formation with or without complications.

Results: 204 patients were included in the study, 163 patients had sleeve gastrectomy (group A) and 41 patients had bypass (group B), the mean age was 35.4 years for group A and $114(69.9 \%)$ of the patients were women, and it was 40.6 years for group B and $28(68.2 \%)$ of patients were women, Gallstones requiring cholecystectomy after corrective weight loss surgery detected in 18 (11\%) of 163 patients had sleeve gastrectomy and $4(9.7 \%)$ of 41 patients had roux en Y gastric bypass, group A patients developed gallstone after 8 months median time, while for group B it was 7 months. No significant difference found between the two groups of patients regarding the symptomatic gall bladder disease.

Conclusions: the incidence of cholelithiasis after corrective weight loss surgery was not so high for these patients and it is not recommended to do prophylactic cholecystectomy.
\end{abstract}

Copy Right, IJAR, 2016,. All rights reserved. 


\section{Introduction:-}

The prevalence of obesity (BMI > 30) in Europe reached around $20 \%$ and in United States became around $30 \%$ that became an epidemic condition with other co-morbidities of metabolic disorders and so was accompanied by life expectancy reduction (1). Corrective weight loss surgery (Bariatric surgery) reduces the body weight and so ameliorates the co-morbidities, while weight reduction conservative management gives less satisfactory results (2), so surgery was recommended for morbid obesity treatment by NIH consensus conference (3).

Laparoscopic sleeve gastrectomy is one of the corrective weight loss surgeries that include removal of the fundus and most of the antrum of the stomach, creating a gastric tube or sleeve which reduces the oral intake (4). This procedure initially introduced as a part of duodenal switch operation, but now become a popular stand-alone procedure for weight loss surgery (5).

About $30 \%$ of the patients who are candidates for bariatric surgery either have undergone a prior cholecystectomy or are found to present gallstones at time of surgery, Gallstone represent the third most common disease observed among obese patients (6). Newly formed gallstones may be diagnosed in $27 \%$ to $43 \%$ of patients who have undergone bariatric surgery within a very short period of time (7), and it was found that the risk of developing gallstones can be as high as $52.8 \%$ in patients undergoing Roux-en-Y gastric bypass who are followed up for 1 year afterwards (8).

Patients with a rapid weight loss, following bariatric surgery, experience increased cholesterol saturation in the bile and also present increased concentrations of mucin in the gallbladder which is the most important pro-nucleating factors (9).

\section{Patients and Methods:-}

This study involved 286 patients followed prospectively were suffering from morbid obesity and underwent laparoscopic sleeve gastrectomy (LSG) and laparoscopic Roux-en-Y Gastric Bypass (LRYGB) in the period from June 2014 till June 2016, data collection began before the operation and continued forward until the latest visit after the operation. Inclusion criteria: Patients with negative abdominal ultrasound preoperatively, patient with at least one year follow up after surgery were included in this study, exclusion criteria: included: patients with gallstone disease preoperatively, history of cholecystectomy, presence of gallbladder polyp on preoperative ultrasound, patients who were lost to follow up after surgery and patients with a previous weight reduction procedure such as intra gastric balloon or gastric band, 82 patients were excluded from the study and 204 patients were included, All patients were discussed concerning the options of LSG and LRYGB and the possible risks of surgery were explained, a written and informed consent taken from all patient preoperatively, Preoperative abdominal ultrasound was performed for all patients to rule out gallstones, sludge or gallbladder polyp. Those with positive findings on ultrasound were discussed for concomitant laparoscopic cholecystectomy. Patients were followed up regularly, every 2-3 months; those with symptoms suggestive of cholelithiasis or its complications underwent further imagining with abdominal ultrasound or magnetic resonance imaging of the abdomen. The patients were divided into two groups for comparison. Group A patients who had (LSG), and group B included the patients who underwent (LRYGB). The primary outcome measure was the formation of symptomatic gallstones or sludge with or without complications. Positive findings according to the ultrasound, or MRI reports were recorded. Symptoms were considered attributable to gallstones or sludge if not clinically explainable by other causes. Patients who had complications such as acute cholecystitis, deranged liver function, acute cholangitis, or biliary pancreatitis at first presentation also were considered as symptomatic in the analysis.

Statistical analysis: Data were collected using Excel for Windows, and analysis was performed with the Scientific Package of the Social Sciences version 22 (SPSS). The chi- square test or Fisher's exact test was used for nominal variables as appropriate. The t-test was used to compare continuous variables. A p value less than 0.05 was considered significant.

\section{Results:-}

During the period from June 2014 till June 2016, 224 patients underwent LSG and 62 patients had LRYGB, after exclusion from the study, 163 group A patients and 41 group B patients were included in the study (table 1). Preoperative cholelithiasis was found in $16(7.1 \%)$ of patients in group A and $5(8 \%)$ of patients in group B, all patients with gallstone disease that diagnosed preoperatively underwent concomitant cholecystectomy, 7 patients 
$(3.1 \%)$ in group A had previous weight reduction procedures (a previous gastric band and a previous intragastric balloon placement), while in group B, 4 (6.4\%) patients had a previous intragastric balloon and all these patients were excluded from the study.

Table 1:- the incidence of cholelithiasis before surgery and exclusions of the patients.

\begin{tabular}{|c|c|c|}
\hline & $\begin{array}{c}\text { Group A } \\
\text { LSG }\end{array}$ & $\begin{array}{c}\text { Group B } \\
\text { LRYGB }\end{array}$ \\
\hline Total number of patients & 224 & 62 \\
\hline Exclusion number $(\%)$ & $61(27.2 \%)$ & $5(3 \%)$ \\
\hline Cholelithiasis on preoperative US & $16(7.1 \%)$ & $1(1.6 \%)$ \\
\hline GB polyp on US & $3(1.3 \%)$ & $4(6.4 \%)$ \\
\hline Previous cholecystectomy & $13(5.8 \%)$ & $4(6.4 \%)$ \\
\hline Previous weight reduction procedures & $7(3.1 \%)$ & $7(11.2 \%)$ \\
\hline Lost to follow up & $22(9.8 \%)$ & 41 \\
\hline
\end{tabular}

The demographics of the patients in the study in both groups are shown in (table 2) the mean age of was 35.4 (18 49 years) for group A, $114(69.9 \%)$ of the patients were women and $49(30.1 \%)$ of the patients were men , whereas it was 40.6 (26 - 54 years) for group B and, $28(68.2 \%)$ of the patients were women and $13(31.8 \%)$ of the patient were men, the Preoperative mean BMI was 50.3 \pm 6.9 (range from 34.6-64.2) in group A patients, and 52.8 \pm 7.4 (range from 41.7 -65.6) in group B patients. The median follow up period was 12 month (range 10 - 14 months) for sleeve group and 16 months (range 12-24 months) in gastric bypass group.

Table 2:- the patient demographics in the study.

\begin{tabular}{|c|c|c|c|}
\hline & & $\begin{array}{c}\text { Group A } \\
(\mathrm{n}=163)\end{array}$ & $\begin{array}{c}\text { Group B } \\
(\mathrm{n}=41)\end{array}$ \\
\hline \multicolumn{2}{|c|}{ Mean age : years (Range) } & $35.4(18-49)$ & $40.6(26-54)$ \\
\hline \multirow[t]{2}{*}{ Gender } & Male & $49(30.1 \%)$ & $13(31.8 \%)$ \\
\hline & Female & $114(69.9 \%)$ & $28(68.2 \%)$ \\
\hline \multicolumn{2}{|c|}{ Preoperative BMI* $\left(\mathrm{Kg} / \mathrm{m}^{2}\right)$} & $50.3 \pm 6.9$ & $52.8 \pm 7.4$ \\
\hline \multicolumn{2}{|c|}{ Median follow up (months) } & 12 & 16 \\
\hline
\end{tabular}

*BMI= body mass index

Symptomatic gallstones requiring cholecystectomy occurred in 18 (11\%) of 163 patients had LSG, and in 4 (9.7 \%) of 41 patients had LRYGB, and this difference was statistically not significant (p. Value >0.05), group A patients developed gallstone after 8 months median time, while for group B it was 7 months and this difference was statistically not significant( p.value $>0.05$ ). Mean BMI at the time of cholecystectomy was $30.5 \pm 6.8 \mathrm{~kg}$ in group A (range from $19.5-47.6 \mathrm{~kg} / \mathrm{m} 2$ ) and $35.3 \pm 5.1 \mathrm{~kg} / \mathrm{m} 2$ (range $26.2-42.8 \mathrm{~kg} / \mathrm{m} 2$ ) in group B (p. Value 0.07 ) table 3.

Table 3:- the comparison of the patients who developed gallstone in group A and B.

\begin{tabular}{|c|c|c|c|}
\hline & & Group A & Group B \\
\hline \multicolumn{2}{|c|}{ Number of patients } & $18(11 \%)$ & $4(9.7 \%)$ \\
\hline \multirow[t]{2}{*}{ Gender } & Male & $4(22.2 \%)$ & $1(25 \%)$ \\
\hline & Female & $14(77.8 \%)$ & $3(75 \%)$ \\
\hline \multicolumn{2}{|c|}{$\begin{array}{l}\text { BMI at the time of Bariatric surgery } \\
\qquad\left(\mathrm{Kg} / \mathrm{m}^{2}\right)\end{array}$} & $50.3 \pm 6.9$ & $52.8 \pm 7.4$ \\
\hline \multicolumn{2}{|c|}{$\begin{array}{l}\text { BMI at the time of cholecystectomy } \\
\qquad\left(\mathrm{Kg} / \mathrm{m}^{2}\right)\end{array}$} & $30.5 \pm 6.8 \mathrm{~kg}$ & $35.3 \pm 5.1$ \\
\hline \multicolumn{2}{|c|}{ Interval (months) } & $8 \pm 3.4$ & $12 \pm 7.2$ \\
\hline
\end{tabular}

All patients who developed symptomatic cholelithiasis underwent laparoscopic cholecystectomy. No patient developed complications requiring re-admission or reoperation after cholecystectomy. No mortality occurred in our patients after cholecystectomy. 


\section{Discussion:-}

Laparoscopic cholecystectomy in the morbidly obese may be associated with increased operative difficulty and morbidity compared with non-obese patients (10). However, the role of prophylactic cholecystectomy at the time of bariatric surgery remains controversial. The fact that pathological evidence of gallbladder diseases has been found in more than $75 \%$ of routinely resected specimens supports those who advocate prophylactic cholecystectomy (11).

On the other hand, the fact that only 7 to $16 \%$ of patients will develop symptomatic gallstones $(12,13)$, and that less than $10 \%$ of patients with negative ultrasound exams require subsequent cholecystectomy (14) does not support performing a prophylactic cholecystectomy. Of our LRYGB group, $9.7 \%$ developed symptomatic cholelithiasis and underwent laparoscopic cholecystectomy, this result is the same in many studies done by Nagem et al (15)and Li et al(16) which reported a rate ranged from 6.7 to $11.8 \%$, theoretically, cholelithiasis is less common after laparoscopic sleeve gastrectomy, because the procedure does not alter the gastrointestinal pathway $(16,17)$.

In our LSG patients, $11 \%$ developed symptomatic gallstones, and this rate was not statistically significant(p. Value $>0.05$ ) from that of LRYGB patients, Li et al (16) also reported no significant difference in the rate for symptomatic and complicated cholelithiasis between LRYGB and LSG group of patients. Although rapid weight loss after bariatric surgery is a risk factor for forming gallstones, we should not neglect that a high number of patients already had gallstone disease or previous cholecystectomy before Bariatric procedure possibly because of obesity factor. In our study we found $5.8 \%$ of sleeve patients and $6.4 \%$ of LRYGB patients, already had their gallbladder removed or had gallstone disease before the operation. This result is similar to that of other studies $(16,18)$. Portenier et al (14) argued that the incidence of delayed cholecystectomy for symptoms or complications of gallstone formation was much lower than the $\geq 30 \%$ reported to develop gallstones and suggested an expectant approach of the asymptomatic cholelithiasis in LRYGB patients. We agree on the point that the percentage of patients developing gallstones is not as high as it was perceived to be.

However, routine preoperative ultrasound screening for gallstones and/or prophylactic cholecystectomy may still be beneficial in LRYGB patients, because access to the biliary tract after LRYGB is challenging (18). In addition, positive gallstones in workups may interfere with diagnosing other reasons when the symptoms are not specific.

These study findings had two important implications. First, because symptomatic cholelithiasis showed no statistical significant compared with gastric bypass; routine prophylactic cholecystectomy is not indicated for these patients. Second, from an etiologic point of view, the traditional belief that hormonal changes and left vagal hepatic branch denervation after gastric bypass result in gallstone formation may no longer hold true because patients who had food going through the usual gastrointestinal transit without duodenal exclusion did not have a lower risk for symptomatic gallstone formation(19,20).

The speed and the degree of weight loss after surgery may be solely related to symptomatic cholelithiasis rather than the choice of the procedure itself (20).

\section{Conclusion:-}

Cholelithiasis was common in our patients before weight reduction surgery. The rate for symptomatic gallstones after surgery was not so high. No significant difference in symptomatic gallstone disease was found between the patients who underwent laparoscopic gastric bypass and those who had sleeve gastrectomy. Routine prophylactic cholecystectomy should not be recommended for these patients.

\section{References:-}

1. H. Mustone, J. Pietila, N. Jaser, et al, preoperative transabdominal ultrasonography prior to laparoscopic Rouxen-Y Gastric Bypass and laparoscopic sleeve gastrectomy in the first 100 operations. Was it beneficial and reliable during the learning curve? Obes Surg, 2012; 416-421.

2. A. Maggard, L.Shugarman, M. Suttorp, et al, Meta-analysis: surgical treatment of obesity, Annals of internal medicine, 2005, 142(7):547-561.

3. NIH conference, gastrointestinal surgery for severe obesity, annals of internal Medicine, 1991; 115:956-961.

4. E. Durmush, G. Ermerak and D. Durmush, short-term outcomes of sleeve gastrectomy for morbid obesity: dose staple line reinforcement matter? Obes Surg, 2014; 24:1109-1116. 
5. S.Aggarwal,A. Prakash, and N. Ramaswamy, outcome of laparoscopic sleeve gastrectomy with and without staple line oversewing in morbidly obese patients: A randomized study, Journal of laparoscopic and advanced surgical techniques, 2013; 23(11):895-899.

6. M. Bastouly, C.Arasaki, J. Ferreira et al, Early changes in postprandial gallbladder emptying in morbidly obese patients undergoing Roux-en-Y gastric bypass: correlation with occurrence of biliary sludge and gallstones, Obes Surg, 2009;19:22-28.

7. C. Oliveira, E. Chaim, B.da Silva, Impact of rapid weight reduction on risk of cholelithiasis after bariatric surgery, Obes Surg. 2003;13(4):625-8.

8. L. Wudel, J.Wright, J. Debelak, et al, Prevention of gallstone formation in morbidly obese patients undergoing rapid weight loss: results of a randomized controlled pilot study, J Surg Res, 2002;102(1):50-56.

9. E. Sioka, D. Zacharoulis, E. Zachari, et al, complicated gallstones after laparoscopic sleeve gastrectomy, Journal of obesity, 2014; 2014:1-5.

10. B. Ammori, A. Vezakis, D. Davides, et al, laparoscopic cholecystectomy in morbidly obese patients, Surg Endosc, 2001;15:1136-1139.

11. M.Fobi, H.Lee, D. Igwe, et al, prophylactic cholecystectomy with gastric bypass operation: incidence of gallbladder disease, Obes Surg, 2002; 12:350-353.

12. L. Villegas, B. Schneider, D. Provost, et al, Is routine cholecystectomy required during laparoscopic gastric bypass? Obes Surg, 2004; 14:206-211.

13. A. Dhabuwala, R. Cannan, and R. Stubbs, improvement in comorbidities following weight loss from gastric bypass surgery, Obes Surg, 2000; 10:428-435.

14. D. Portenier, J. Grant, H. Blackwood, et al, expected management of the asymptomatic gallbladder at Roux-enY Gastric bypass, Surg Obes Relat Dis, 2007;3:476-479.

15. R. Nagem and A. Da-Sliva, Cholecystolithiasis after Gastric Bypass: A Clinical, Biochemical, and Ultrasonographic 3-year Follow-up Study, Obes Surg,2012; 22:1594-1599.

16. V. Li, N. Pulido, P. Suartez, et al, symptomatic gallstones after sleeve gastrectomy, Surg Endosc, 2009; 23:2488-2492.

17. R. Kiewiet, M. Durian, M. Leesum, et al, gallstone formation after weight loss following gastric banding in morbidly obese Dutch patients, Obes Surg, 2006;16:592-596.

18. M. Falcao, M. Campos, M. Galvato, et al, transgastric endoscopic retrograde cholangiopancreatography for the management of biliary tract disease after Roux-en-Y gastric bypass treatment for obesity, Obes Surg,2012;22:872-876.

19. I. Samuel, E. Mason, K. Renquist, et al, An 18 year report from the international bariatric surgery registry, Am J Surg, 2006; 192:657-662.

20. V.Li, N. Pulido, P. Fajwaks,et al, Predictors of gallstone formation after bariatric surgery: a multivariate analysis of risk factors comparing gastric bypass, gastric banding, and sleeve gastrectomy, Surg Endosc,2009;23: 1640-1644. 